\title{
Review Article \\ Prevalence of Cardiovascular Complications in Malaria: A Systematic Review and Meta-Analysis
}

\author{
Anna Engell Holm, ${ }^{1,2}$ Laura C. Gomes, ${ }^{1}$ Claudio Romero Farias Marinho, ${ }^{3}$ Odilson M. Silvestre, ${ }^{1}$ Lasse S. Vestergaard, ${ }^{4}$ \\ Tor Biering-Sørensen, ${ }^{2,5}$ and Philip Brainin ${ }^{1,2 *}$ \\ ${ }^{1}$ Federal University of Acre, Campus Floresta, Cruzeiro do Sul, Acre, Brazil; ${ }^{2}$ Department of Cardiology, Herlev-Gentofte Hospital, Hellerup, \\ Denmark; ${ }^{3}$ Department of Parasitology, Institute of Biomedical Sciences, University of São Paulo, São Paulo, Brazil; ${ }^{4}$ National Malaria Reference \\ Laboratory, Department of Bacteria, Parasites and Fungi, Statens Serum Institut, Copenhagen, Denmark; ${ }^{5}$ Faculty of Biomedical Sciences,
} Copenhagen University, Copenhagen, Denmark

\begin{abstract}
Recent studies have suggested that malaria may affect the cardiovascular system. The aim of this systematic review and meta-analysis was to determine the prevalence of cardiovascular complications in symptomatic malaria patients. We searched databases such as Pubmed, Embase, Cochrane, and Web of Science (January 1950-April 2020) for studies reporting on cardiovascular complications in adults and children with malaria. Cardiovascular complications were defined as abnormalities in electrocardiogram (ECG), cardiac biomarkers, and echocardiography on admission or during outpatient examination. Studies of patients with known heart disease or cardiovascular evaluation performed after the start of intravenous antimalarial medication were excluded. The study was registered in International Prospective Register of Systematic Reviews (PROSPERO) (No.: CRD42020167672). The literature search yielded 1,243 studies, and a total of 43 studies with symptomatic malaria patients were included. Clinical studies $(n=12$ adults; $n=5$ children) comprised 3,117 patients, of which a majority had Plasmodium falciparum $(n=15)$ and were diagnosed with severe malaria $(n=13)$. In random-effects models of adults, the pooled prevalence estimate for any cardiovascular complication was $7 \%(95 \% \mathrm{Cl}: 5-9)$. No meta-analysis was conducted in children, but the range of abnormal ECG was $0-8 \%$, cardiac biomarkers $0-57 \%$, and echocardiography $4-9 \%$. We analyzed 33 cases ( $n=10$ postmortem), in which the most common cardiovascular pathologies were myocarditis and acute coronary syndrome. All histopathological studies found evidence of parasitized red blood cells in the myocardium. Cardiovascular complications are not uncommon in symptomatic adults and children with malaria. Additional studies investigating malaria and cardiovascular disease are encouraged.
\end{abstract}

\section{INTRODUCTION}

Despite progress in global control and elimination efforts, malaria remains a widespread parasitic disease. In 2018, approximately 228 million cases and 405,000 deaths worldwide were attributed to malaria. ${ }^{1}$ In low- and middle-income countries, malaria remains a major burden on local health services, demanding frequent hospitalizations and adding to morbidity. ${ }^{2}$ Although malaria mortality has decreased markedly since 2000, a concomitant increase in cardiovascular deaths has been reported in malaria-endemic areas., ${ }^{3,4}$ Despite links between parasitic infections and cardiovascular disease have been demonstrated previously, ${ }^{5}$ the relationship between malaria and cardiovascular illness is poorly investigated.

Among the human malaria species, infection with Plasmodium falciparum is considered the most lethal. Uncomplicated malaria may arise as a result of $P$. falciparum infection, which is often characterized by periodic fever, body ache, and diarrhea; yet, the disease may also develop into its complicated forms often designated as severe or complicated malaria. ${ }^{6} \mathrm{~A}$ known complication to severe malaria is rapid fluid depletion, which may affect the cardiac output. ${ }^{7}$ Cerebral malaria and severe malarial anemia are well-characterized forms of severe disease caused by $P$. falciparum. This results mostly from a combination of events involving structural modifications and expression of parasite adhesion molecules in the infected erythrocyte that promote their accumulation in specific organs such as the brain and placenta, ${ }^{8,9}$ triggering local *Address correspondence to Philip Brainin, Federal University of
Acre, Campus Floresta, Rua do Embira 341, Bairro Joao Alves,
Cruzeiro do Sul, Acre 69980-000, Brazil. E-mail: denlillefilur@ hotmail.com inflammation that participates in parasite clearance with a downside contribution to the pathological outcomes of severe malaria. ${ }^{10}$ The cytoadhesive properties of Plasmodium vivax remain less well described. ${ }^{11}$ Although no studies have demonstrated cytoadherence of infected erythrocytes to the myocardial endothelium, autopsy data suggest that infected erythrocytes may block myocardial capillaries ${ }^{12}$ consequently, leading to mechanical blood flow obstruction and potential myocardial ischemia. ${ }^{13,14}$ Other proposed mechanisms involve release of parasite toxins causing apoptosis of cardiac myocytes $^{15}$ and impairment of vascular endothelial function, resulting in upregulation of pro-inflammatory cytokines and myocardial dysfunction. ${ }^{16-18}$ At present, the pathophysiology between malaria and cardiac illness is not entirely understood, and an appraisal of the most common cardiovascular complications in the acute setting of malaria may prove beneficial to improve both treatment and prognosis of patients. Therefore, the aim of this systematic review and meta-analysis was to provide an estimate of the prevalence of the most commonly reported cardiovascular complications in malaria.

\section{METHODS}

We included clinical studies, case reports, and series of cases addressing cardiovascular complications caused by malaria infection. Reporting was performed in accordance with Preferred Reporting Items for Systematic Reviews and Meta-Analyses. This systematic review was registered at the International Prospective Register of Systematic Reviews (PROSPERO; No.: CRD42020167672).

Selection criteria. We included full-text reports on patients in all ages who were positive for malaria infection with 
P. falciparum, Plasmodium ovale, P. vivax, Plasmodium malariae, or Plasmodium knowlesi verified by microscopy of Giemsa-stained peripheral blood films, quantitative buffy coat, rapid diagnostic tests, or polymerase chain reaction. Other types of Plasmodium were excluded. We included patients with both mild and severe infections as defined by the WHO. ${ }^{19}$ Cardiovascular complications had to be reported on admission and involved abnormalities in electrocardiograms (ECG), cardiac biomarkers, and echocardiography. Postmortem studies were only required to report autopsy findings. We excluded articles in languages other than English, animal and in vitro studies, vertical transmission and studies of patients with concomitant parasite infections, congenital heart disease, heart transplant, or known heart disease at baseline. We also excluded reports on patients who underwent cardiovascular evaluation after treatment start of intravenous antimalarial medication, as this may induce iatrogenic cardiac complications. $^{20,21}$

Search strategy. We searched in PubMed, Embase, Cochrane, and Web of Science from January 1950 to April 2020. We applied a broad search string to increase the sensitivity of the search, and some of the following search terms were used: "Malaria" OR " $P$. falciparum" OR " $P$. vivax" AND "Heart Disease" OR "Cardiovascular." The full search strings for each of the databases are displayed in Supplemental Table 1. We also examined the reference lists of all included articles to identify additional articles fulfilling our inclusion/ exclusion criteria.

Data collection. The literature search and screening of titles and abstracts were performed by two independent reviewers (A. E. H. and P. B.). After this step, duplicates were removed, and full-text reports were assessed for eligibility by the same reviewers. Any disagreements were resolved by consensus. Data extraction was independently performed by A. E. H. and L. C. G. For all studies, we assessed country, sample size, clinical characteristics (age and gender), cardiovascular risk factors, diagnostic method, species, severity, parasite density, and data on cardiovascular complications at admission. According to guidelines in cardiovascular medicine, we extracted data on common alterations related to myocardial damage, involving ECG changes, ${ }^{22,23}$ biomarkers, ${ }^{24}$ and echocardiography. ${ }^{25}$ For ECG, this involved deviations from sinus-rhythm and ST-segment changes. Abnormal levels of cardiac biomarkers (i.e., troponins, $\mathrm{N}$-terminal pro-brain natriuretic peptide) and echocardiographic parameters (i.e., left ventricular ejection fraction [LVEF]) ${ }^{25}$ were assessed according to internationally recognized reference ranges. ${ }^{26,27}$ When studies presented individual person data, we extracted the frequency of abnormal cardiac parameters. When studies compared cardiovascular complications in malaria cases versus controls, these data were also extracted. For clinical studies (longitudinal, cross-sectional, and casecontrol), we extracted data on cardiovascular mortality when available. In case reports and series of cases, we also obtained information on histopathology where available. Studies with pediatric populations were defined as $\leq 17$ years old and adult populations $\geq 18$ years old.

Bias assessment. We used the study quality assessment tool from the National Heart, Lung, and Blood Institute for observational cohort and cross-sectional studies. ${ }^{28}$ Two investigators (A. E. H. and L. C. G.) applied this tool to clinical studies to assess risk of bias. The assessment yielded an overall good quality of included clinical studies (Supplemental Table 2).

Statistics. We applied a random-effects model for pooling proportions of cardiovascular complications in adults. ${ }^{29}$ This was performed using the metaprop command in STATA (StataCorp, College Station, TX). Because of a limited number of studies, no meta-analysis was conducted in children. The variation across studies caused by heterogeneity was assessed by the $I^{2}$ value. However, for abnormal echocardiography in adults, $l^{2}$ was not assessed because of few included studies. A forest plot was constructed showing the prevalence of cardiovascular complications among all studies together with the pooled measure. Subgroup meta-analyses were conducted for each of the cardiovascular complications (ECG, biomarkers, and echocardiography). In addition, we conducted meta-regression models to determine if country, year, species, and severity affected our results. We assessed potential publication bias using Egger's test in each subgroup. ${ }^{30}$ We considered reported $P$-values < 0.05 as significant. All statistical analyses were performed using STATA (version 14.2, College Station, TX).

\section{RESULTS}

The literature search and identification of records in bibliographies yielded 1,243 studies, of which 1,087 were excluded based on title and abstract. A total of 156 studies were assessed in full text, of which 43 fulfilled the inclusion criteria. The studies were divided in two major categories, the first involving 17 clinical studies ${ }^{31-47}$ and the second 26 case reports and series of cases ${ }^{48-73}$ (Figure 1).

Clinical studies. Clinical studies of adults $(n=12)$ and children $(n=5)$ were conducted from 1992 to 2020 and involved a total of 3,117 patients $(n=2,403$ adults and $n=714$ children), of which one study ${ }^{57}$ accounted for $49 \%$ of all patients $(n=1,531)$ (Table 1$)$.

Fifteen studies assessed infection with $P$. falciparum, of which a majority examined severe malaria cases $(n=$ 13). ${ }^{31,32,34-36,39,41-47}$ Studies were conducted in Southeast Asia $(n=6),{ }^{31,32,34,37,38,41}$ Germany $(n=5),{ }^{35,36,39,40,42}$ Africa $(n=5),{ }^{43-47}$ and South America $(n=1) .{ }^{33}$ The most commonly reported cardiovascular parameters are displayed in Table 2.

Adults. All studies but one involved hospitalized patients ${ }^{33}$ (all studies: age 18-70 years; 33\% female; median parasite density 30,295/ $\mathrm{LL}$ ). In subgroup meta-analyses, the pooled prevalence estimate for abnormal ECG was 7\% $(95 \% \mathrm{Cl}$ : 3-10) $(n=7),{ }^{32,33,37,38,40-42}$ cardiac biomarkers $8 \%(95 \%$ Cl: 5-12) $(n=6),{ }^{34,36,38-41}$ and echocardiography $17 \%(95 \%$ Cl: $11-23)(n=3)^{32,34,42}$ (Figure 2). The overall pooled prevalence estimate for cardiac complications was 7\% (95\% Cl: $5-9)$. Considerable heterogeneity was present $\left(l^{2}=0.90\right)$. In metaregression models, the presence of severe malaria cases in the included studies significantly affected the prevalence estimate of cardiac biomarkers $(P=0.03)$. Country, year, and species did not affect the results $(P<0.05$ for all; Supplemental Table 3). Egger's test indicated publication bias among studies reporting on ECG $(P=0.016)$ and biomarkers $(P=0.024)$ but not in the echocardiography subgroup $(P=0.87)$. Two studies ${ }^{34,42}$ examined patients at follow-up, of which only one found persistent echocardiographic alterations. ${ }^{34}$ Details are displayed in Supplemental Table 4.

Children. All studies were conducted on $P$. falciparum in Africa (age 3 months-10 years; 52\% female; median parasite 


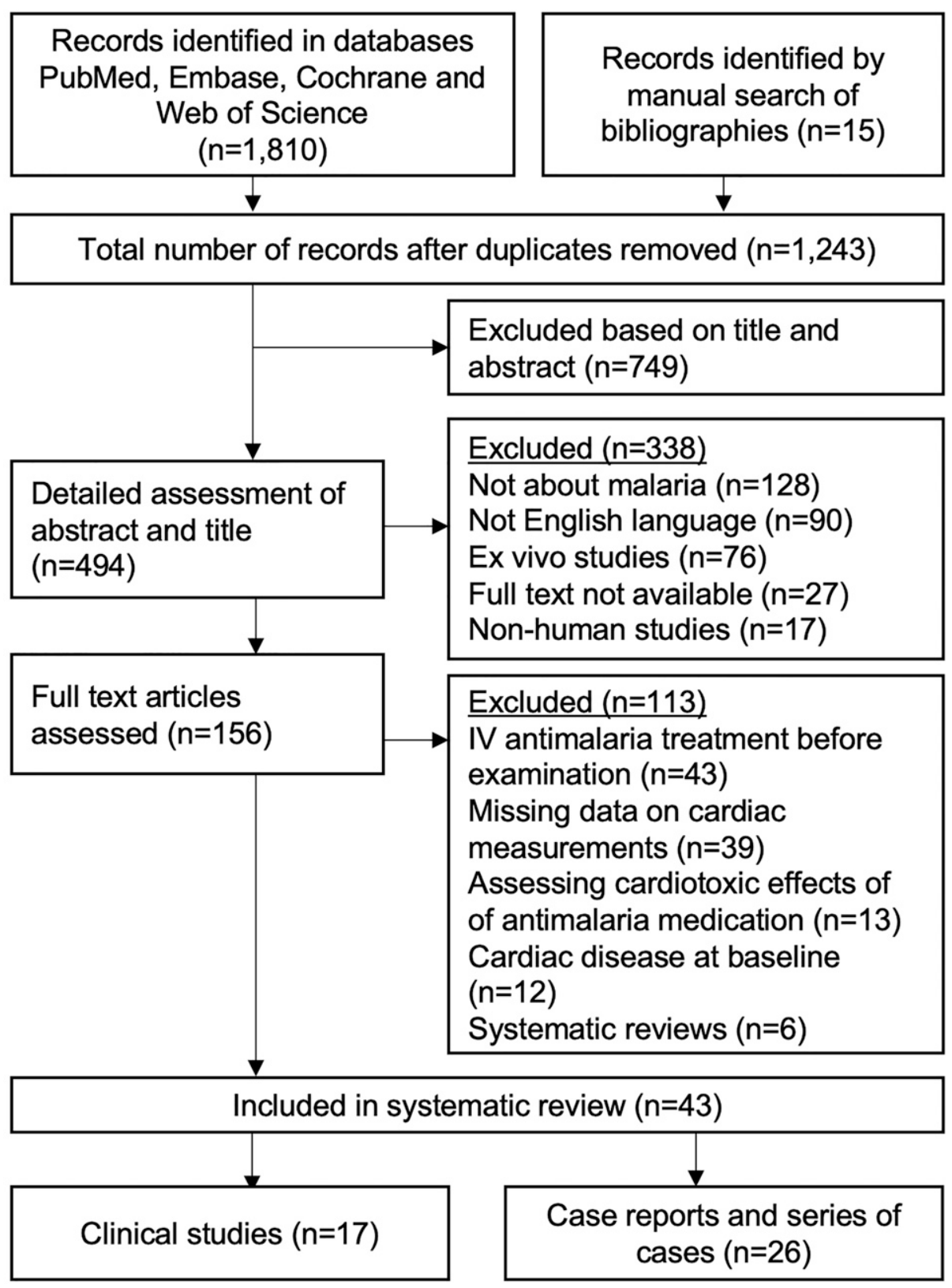

FIGURE 1. Flowchart. Flowchart of included studies in the systematic review and meta-analysis.

density $35,384 / \mu \mathrm{L})$. Only one study reported ECG abnormalities with a frequency of $0 \%,{ }^{44}$ whereas four studies reported abnormal cardiac biomarkers ranging from $0 \%$ to $58 \%$. $^{39,43,44,46}$ The frequency of abnormal echocardiography ranged from $4 \%$ to $9 \%(n=4) .{ }^{43-46}$ One study ${ }^{43}$ found that biomarkers and echocardiography had normalized after 42 days of follow-up. Additional details are found in Supplemental Table 5.

Case reports and series of cases (Figure 3). We analyzed 33 case reports and series of cases ( $n=29$ adults; $n=4$ children; Figure 2) from 1954 to 2019 (age range 1-6 years; 36\% female). A majority of infections were caused by $P$. falciparum $(n=18)$ and $P$. vivax $(n=12)$ (Supplemental Table 6).

Adults. Abnormalities in ECG were present in $55 \%$ of cases, ${ }^{48,50,53,56,58,60,65-67,69,70}$ whereas $14 \%$ had increased troponins (TnT/Tnl). ${ }^{60,66,67,70}$ Five reports found decreased LVEF. ${ }^{53,56,58,68,71}$ However, in four reports, ${ }^{56,58,68,71}$ this normalized at follow-up (range 5-120 days). Most patients were diagnosed with myocarditis $(n=9)^{53,56-60,68,70,71}$ and acute coronary syndrome $(n=4) .{ }^{48,49,66,69}$

Children. All cases were hospitalized, ${ }^{54,61,63}$ of which three ${ }^{61,63}$ had acute heart failure, as determined by the LVEF range $22-35 \%$, and one had myocarditis. ${ }^{54}$ In two cases, the LVEF was normal at follow-up. ${ }^{54,61}$

Postmortem. Ten cases were analyzed ( $n=9 P$. falciparum, $n=1$ P. knowlesi) ${ }^{51,52,55,64,72}$ (Supplemental Table 6). All studies reported inflammation and infiltrates of parasitized red blood cells in the myocardium and cardiac blood vessels.

\section{DISCUSSION}

Cardiovascular complications in malaria have been reported since the beginning of the nineteenth century, ${ }^{74}$ but 
TABLE 1

Frequency of cardiovascular complications in clinical studies according to adult and pediatric populations

\begin{tabular}{|c|c|c|c|c|c|c|c|c|c|}
\hline \multirow[b]{2}{*}{ Author, year } & \multirow[b]{2}{*}{ Country } & \multirow[b]{2}{*}{ Sample size } & \multirow[b]{2}{*}{$\mathrm{SM}, n(\%)$} & \multirow[b]{2}{*}{ Species } & \multirow[b]{2}{*}{ Diagnosis } & \multirow[b]{2}{*}{ Population } & \multicolumn{3}{|c|}{ Frequency of abnormal cardiac parameters } \\
\hline & & & & & & & Electrocardiogram, $n(\%)$ & Biomarkers, $n(\%)$ & Echocardiography, $n(\%)$ \\
\hline \multicolumn{10}{|c|}{ Adult populations $(n=12)$} \\
\hline Bhardwaj $^{31}$ & India & 74 & 7 (9) & Pf & PBF & Hospitalized & N/A & + & N/A \\
\hline Ray $^{32}$ & India & 27 & $27(100)$ & $\mathrm{Pf}, \mathrm{Pv}$ & PBF & Hospitalized & $4(15)$ & $\mathrm{N} / \mathrm{A}$ & $5(19)$ \\
\hline Alencar-Filho ${ }^{33}$ & Brazil & 26 & 0 & $\mathrm{Pv}$ & PBF & Outpatient & N/A & + & + \\
\hline Nayak $^{34}$ & India & 100 & $100(100)$ & $\mathrm{Pf}, \mathrm{Pv}$ & PBF & Hospitalized & $9(9)$ & $14(14)$ & $17(17)$ \\
\hline Stauga ${ }^{35}$ & Germany & 79 & $12(15)$ & $\mathrm{Pf}$ & PBF & Hospitalized & N/A & + & N/A \\
\hline $\mathrm{Herr}^{36}$ & Germany & 28 & $7(25)$ & Pf & PBF & Hospitalized & N/A & $7(25)$ & N/A \\
\hline Mehmood $^{37}$ & Pakistan & 97 & 0 & Pv & PBF & Hospitalized & $1(1)$ & N/A & N/A \\
\hline Jain $^{38}$ & India & 1,531 & 0 & Pf, Pv & QBF & Hospitalized & $22(1)$ & $22(1)$ & N/A \\
\hline Ehrhardt ${ }^{39}$ & Germany & 63 & $11(17)$ & $\mathrm{Pf}$ & N/A & Hospitalized & N/A & $28(44)$ & N/A \\
\hline Günther ${ }^{40}$ & Germany & 161 & 0 & Pf & PBF & N/A & $23(14)$ & $1(1)$ & N/A \\
\hline Mohapatra $^{41}$ & India & 195 & $110(100)$ & Pf & PBF & Hospitalized & $12(6)$ & $13(7)$ & N/A \\
\hline Franzen $^{42}$ & Germany & 22 & 0 & $\mathrm{Pf}, \mathrm{Pv}$ & PBF & Hospitalized & $5(23)$ & N/A & $3(14)$ \\
\hline \multicolumn{10}{|c|}{ Pediatric populations $(n=5)$} \\
\hline Nguah $^{43}$ & Ghana & 183 & $183(100)$ & Pf & PBF & Hospitalized & N/A & $106(58)$ & + \\
\hline Mocumbi $^{44}$ & Mozambique & 45 & $18(40)$ & Pf & PBF & Hospitalized & 0 & 0 & $2(4)$ \\
\hline Murphy ${ }^{45}$ & Uganda & 33 & $17(53)$ & $\mathrm{Pf}$ & PBF & Hospitalized & N/A & N/A & $3(9)$ \\
\hline Janka ${ }^{46}$ & Mali & 53 & $53(100)$ & Pf & PBF & Hospitalized & N/A & + & + \\
\hline Ehrhardt $^{47}$ & Ghana & 400 & $200(50)$ & Pf & PBF & Hospitalized & N/A & $226(57)$ & N/A \\
\hline
\end{tabular}

available, but data for malaria cases were significantly different compared with controls.

no systematic reviews have assessed this topic. This study had three principal findings. First, in clinical studies of symptomatic adults with malaria, the pooled prevalence estimate of cardiovascular complications was $7 \%(95 \% \mathrm{Cl}$ : 5-9). Second, in case reports and series of cases, the most common cardiac pathologies were myocarditis and acute coronary syndrome. Third, significant heterogeneity was present among studies reporting on cardiovascular complications in malaria patients.

Infection with Plasmodium may result in a variety of symptoms, ranging from uncomplicated disease to severe symptoms and even death. Many factors influence the likelihood of progression into severe symptoms, including malaria species, age, and host immunity. ${ }^{75}$ During the course of severe malaria, the myocardium appears to be a heavily parasitized organ. ${ }^{76,77}$ This is in line with the post-mortem cases we assessed, which all demonstrated parasitized erythrocytes in the myocardium. ${ }^{51,52,55,64,72}$ After successful invasion of the erythrocyte, $P$. falciparum modifies the cytoadhesive properties of the erythrocyte. ${ }^{9}$ This may allow the accumulation of infected erythrocytes in the myocardial capillaries, leading to mechanical obstruction, reduced tissue perfusion, and disease. ${ }^{13,14}$ Although less common, studies have also demonstrated how $P$. vivax-infected erythrocytes also may adhere to the endothelium. ${ }^{11,13,78}$ Likely, accumulation in the capillaries and microcirculation in the myocardium may result in regional dysfunction, leading to pathological alterations in ECG, biomarkers, and echocardiographic parameters. Those are the same parameters that we found affected in a majority of clinical studies of $P$. falciparum patients on admission. ${ }^{31,32,34-36,39-47,57}$

Although the definition of severe malaria includes cardiovascular collapse, this commonly refers to algid malaria, ${ }^{79}$ a state of circulatory shock accompanied by bacteremia. As stated by the WHO, the presence of myocardial dysfunction and left ventricular impairment is considered very rare in severe malaria. ${ }^{7}$ By contrast, we found that in studies with severe $P$. falciparum, cardiovascular complications were frequently reported on admission. The most recurring pathologies were ST-segment changes and elevated creatine

TABLE 2

Most commonly reported cardiovascular complications in clinical studies

\begin{tabular}{|c|c|c|}
\hline & Adult population & Pediatric population \\
\hline Electrocardiogram & $\begin{array}{l}\text { Nonspecific ST changes }(n=4) \\
\text { Sinus tachycardia }(n=2) \\
\text { First degree AV-block }(n=2) \\
\text { Sinus bradycardia }(n=2)\end{array}$ & Arrhythmia of any kind $(n=1)$ \\
\hline Cardiac biomarkers & $\begin{array}{l}\text { Creatine kinase MB }(n=6) \\
\text { NT-proBNP }(n=6) \\
\text { Troponin }(\operatorname{TnT} / \operatorname{Tnl})(n=5) \\
\text { Myoglobin }(n=4) \\
\text { Cystatin C }(n=3) \\
\text { H-type fatty acid binding protein }(n=2) \\
\text { MR-proANP }(n=1)\end{array}$ & $\begin{array}{l}\text { Troponin }(\operatorname{TnT} / \mathrm{Tnl})(n=2) \\
\text { NT-proBNP }(n=2) \\
\text { Creatine kinase MB }(n=1) \\
\text { Myoglobin }(n=1) \\
\text { Cystatin C }(n=1) \\
\text { H-type fatty acid binding protein }(n=1)\end{array}$ \\
\hline Echocardiography & $\begin{array}{l}\text { LV ejection fraction }(n=4) \\
\text { LV end-diastolic dimension }(n=3) \\
\text { Diastolic dysfunction }(n=2) \\
\text { LV end-systolic dimension }(n=1)\end{array}$ & $\begin{array}{l}\text { LV ejection fraction }(n=1) \\
\text { LV end-diastolic dimension }(n=1)\end{array}$ \\
\hline
\end{tabular}


Study

Electrocardiogram

Ray, 2017 (32)

Nayak, 2013 (34)

Mehmood, 2011 (37)

Jain, 2010 (38)

Günther, 2003 (40)

Mohapatra, 2000 (41)

Franzen, 1992 (42)

Subtotal $\left(I^{\wedge} 2=86.46 \%, p=0.00\right)$

Biomarkers

Nayak, 2013 (34)

Herr, 2011 (36)

Jain, 2010 (38)

Ehrhardt, 2004 (39)

Günther, 2003 (40)

Mohapatra, 2000 (41)

Subtotal $\left(I^{\wedge} 2=93.64 \%, p=0.00\right)$

Echocardiography

Ray, 2017 (32)

Nayak, 2013 (34)

Franzen, 1992 (42)

Subtotal

Heterogeneity between groups: $p=0.016$

Overall $\left(I^{\wedge} 2=89.83 \%, p=0.00\right)$;

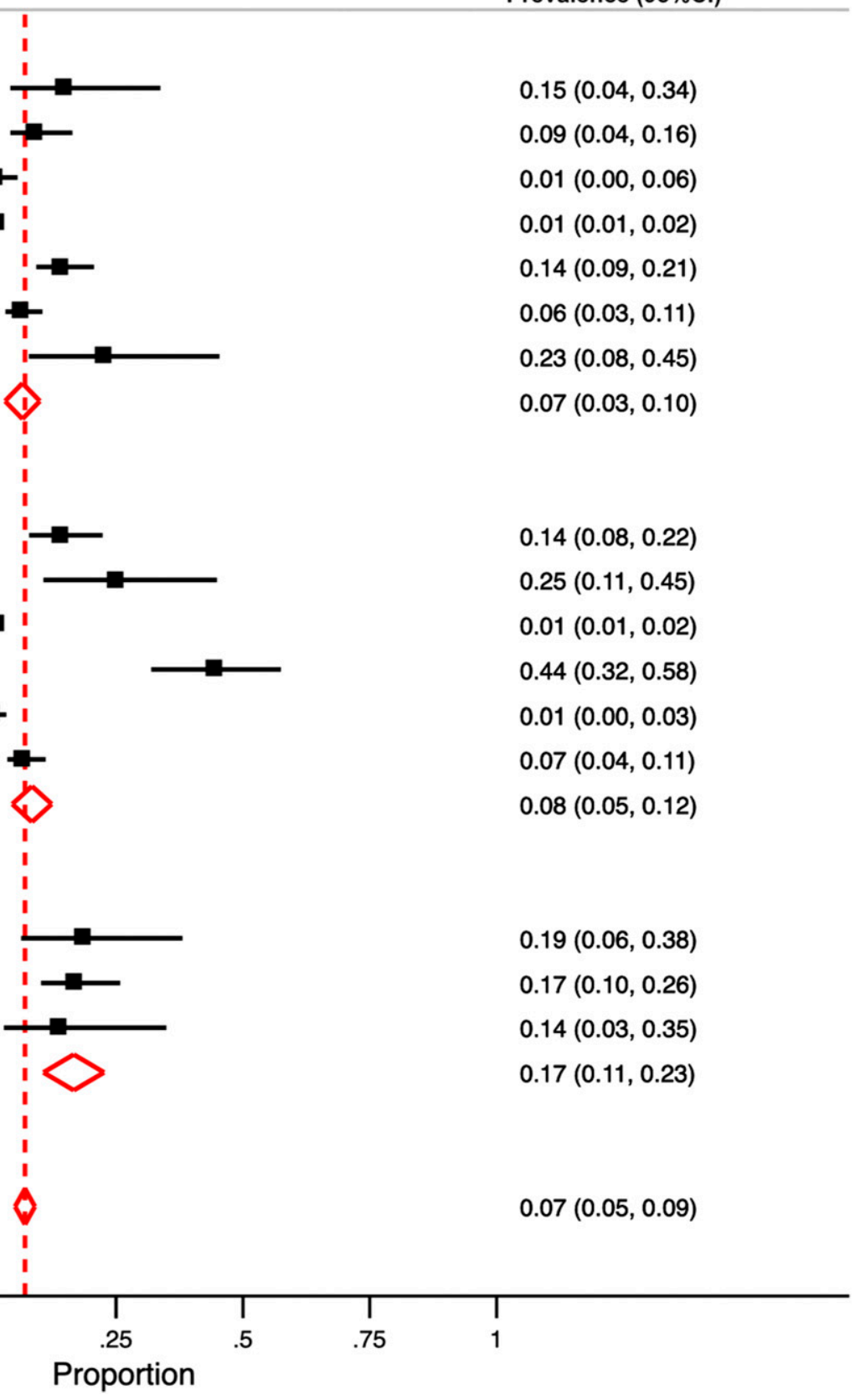

FIGURE 2. Meta-analysis of cardiovascular complications. Forest plot for random-effects meta-analysis displaying the proportions and pooled estimate for having cardiovascular complications. Subgroup meta-analysss are conducted for abnormal electrocardiogram, biomarkers, and echocardiography. Solid black lines indicate the prevalence estimate for each included study. Red dashed line indicates the pooled prevalence estimate for any cardiovascular complication. This figure appears in color at www.ajtmh.org.

kinase myocardial band (CK-MB) and, specifically, two studies demonstrated impaired left ventricular function at admission (Table 2). ${ }^{32,34}$ Considering that malaria may rapidly progress to complications and death without appropriate treatment, an appraisal of cardiovascular parameters in the clinical setting could potentially serve as early indicators of disease severity. In addition, it is known that multiple affected organ systems during the course of severe malaria are associated with mortality. ${ }^{80}$ As only three studies assessed cardiac parameters prospectively, and given that they presented inconsistent data from small populations, ${ }^{34,42,43}$ it remains uncertain if cardiac complications may proceed beyond the acute setting, and also may contribute to development of long-term heart disease, such as heart failure. ${ }^{81}$

In the two studies that examined $P$. vivax patients, ${ }^{33,37}$ cardiovascular complications were also reported. Unlike $P$. falciparum, $P$. vivax seldom develops hyperparasitemia, but may lead to a greater inflammatory response and endothelial activation per parasite than $P$. falciparum. ${ }^{82}$ Recently, it was proposed that $P$. vivax inflammation may be responsible for pulmonary injury. ${ }^{83}$ One-third of all analyzed case reports had 


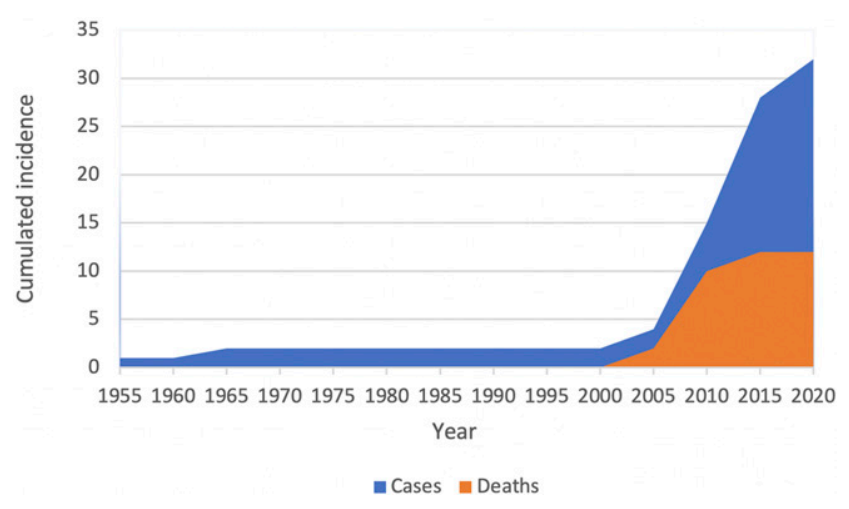

Figure 3. Cumulated incidence of malaria cases and cardiovascular death. Cumulated reporting of malaria cases (blue) and cardiovascular death (orange) according to time period. Data are from the included case reports and series of cases. This figure appears in color at www.ajtmh.org.

P. vivax, of which $75 \%$ were diagnosed with myocarditis and/ or pericarditis. Several of the pro-inflammatory cytokines associated with $P$. vivax, ${ }^{84,85}$ particularly tumour necrosis factor-alfa, are known to potentiate myocardial inflammation. ${ }^{86}$ Albeit speculative, $P$. vivax may potentially be related to active inflammatory damage of the myocardium. However, this should be explored in future clinical studies.

Considerable heterogeneity was observed in the reporting of cardiac complications $\left(I^{2}=0.90\right)$, and only a minority of studies reported on all three parameters (ECG, biomarkers, and echocardiography). ${ }^{34,44}$ We found that studies included a wide range of biomarkers (Table 2), and that several studies lacked to provide cutoff values for categorizing biomarkers as elevated. ${ }^{31,33-36,38,43}$ Only four of seven studies with echocardiography reported on the LVEF, ${ }^{33,34,42,43}$ which is a key ultrasonographic parameter. Also, studies failed to provide the diagnostic method for malaria $^{39}$ and parasite density. ${ }^{37,38,40,41,44,45}$ The heterogeneity and lack of standardized reporting of cardiovascular complications in malaria witness that no guidelines nor prior large-scale clinical studies have investigated this topic. In comparison, features of respiratory impairment in malaria have been addressed in clinical studies ${ }^{83}$ and reports by the WHO, ${ }^{7,87-89}$ yielding a systematic approach to describe pulmonary changes. A similar systematic approach to cardiovascular complications in malaria is encouraged.

Limitations. We only included studies reporting on cardiovascular complications, which could lead to an overestimation of the frequency of these parameters. Publication bias may have affected our results, as severe cases of malaria are more likely to be published. This is supported by Egger's tests, which indicated publication bias in studies reporting on ECG and cardiac biomarkers. Lack of systematic reporting, different objectives, and use of different control groups (healthy $^{31,33,35,36}$ versus uncomplicated malaria ${ }^{32,39,41}$ ) may have affected our results. Malaria is not a uniform disease; it may therefore be controversial to include several species in the same study. Severe malaria is more common in children $<5$ years old, ${ }^{90}$ indicating pathophysiological differences between adult and pediatric populations. Despite this, cardiovascular complications, and also left ventricular impairment, ${ }^{43}$ were present at almost the same frequency in children as for adults. Five studies reported on imported malaria in
Germany, ${ }^{35,36,39,40,42}$ which constitutes a major limitation, as immunity profiles ${ }^{91}$ may be different. Considering that the cytoadhesive properties of $P$. falciparum may differ according to geographical regions, ${ }^{92}$ and the assessed studies came from Africa, Asia, and South America, this could potentially affect cardiovascular impairment. Assessment of cardiac biomarkers and echocardiography requires expensive laboratory facilities and equipment, which may be limited in malaria-endemic and resource-constrained settings. Underreporting, misdiagnosis, and incorrect data may have influenced our analyses. Secondary complications to severe malaria disease, including anemia, renal failure, and acute respiratory distress syndrome, could have been misdiagnosed or contributed to cardiovascular disease. Only a minority of clinical studies reported on cardiovascular mortality; hence, we could not assess this. Recent studies have hypothesized that malaria through various pathways may be linked to hypertension, which is also a major risk factor for cardiac disease. ${ }^{93-95}$ Unfortunately, only a minority of studies reported on hypertension. ${ }^{32-34,36,37,42,44}$

\section{CONCLUSION}

To the authors' knowledge, this is the first systematic review to assess cardiovascular complications in malaria. The key findings are that abnormalities in ECG, cardiac biomarkers, and echocardiography were not uncommon in children and adults with malaria. However, great heterogeneity was present in the assessed studies, indicating a need for standardized protocols with adequate data registering, streamlining of methods and measurements to better characterize the relationship between malaria and the cardiovascular system.

Received November 4, 2020. Accepted for publication January 2, 2021.

Published online March 15, 2021.

Note: Supplemental tables appear at www.ajtmh.org.

Financial support: P. B. was funded by a research grant from the Independent Research Fund Denmark (0129-00003B).

Authors' addresses: Anna Engell Holm, Herlev-Gentofte Hospital, Hellerup, Denmark and Federal University of Acre, Acre, Brazil, E-mail: annaengellholm@gmail.com. Laura C. Gomes, Federal University of Acre and University of São Paulo, São Paulo, Brazil, E-mail: lauracgomes@gmail.com. Claudio Romero Farias Marinho, University of São Paulo, São Paulo, Brazil, E-mail: crfmarinho@gmail.com. Odilson M. SilvestrȩFederal University of Acre, Acre, Brazil, E-mail: odilsonms@gmail.com. Lasse S. Vestergaard, Statens Serum Institut, Copenhagen, Denmark, E-mail: lav@ssi.dk. Tor Biering-Sørensen, Herlev-Gentofte Hospital, Hellerup, Denmark, and Copenhagen University, Copenhagen, Denmark, E-mail: tor.biering@gmail.com. Philip Brainin, Herlev-Gentofte Hospital, Hellerup, Denmark and Federal University of Acre, Brazil, E-mail: denlillefilur@hotmail.com.

\section{REFERENCES}

1. World Health Organization, 2019. World Malaria Report 2019. Geneva, Switzerland: WHO.

2. Meara WPO, Noor A, Gatakaa H, Tsofa B, Mckenzie FE, Marsh K, 2019. The impact of primary health care on malaria morbidity defining access by disease burden. Eur PMC Funders Gr 14: 29-35.

3. Ruan Y, Guo Y, Zheng Y, Huang Z, Sun S, Kowal P, Shi Y, Wu F, 2018. Cardiovascular disease (CVD) and associated risk factors among older adults in six low-and middle-income countries: results from SAGE wave 1. BMC Public Health 18: 778. 
4. Owolabi M, Miranda JJ, Yaria J, Ovbiagele B, 2016. Controlling cardiovascular diseases in low and middle income countries by placing proof in pragmatism. BMJ Glob Heal 1: e000105.

5. Blum JA, Zellweger MJ, Burri C, Hatz C, 2008. Cardiac involvement in African and American trypanosomiasis. Lancet Infect Dis 8: 631-641.

6. Ashley EA, Pyae Phyo A, Woodrow CJ, 2018. Malaria. Lancet 391: 1608-1621.

7. World Health Organization, 2014. Severe malaria. Trop Med Int Heal 19: 7-131.

8. Craig AG, Khairul MFM, Patil PR, 2012. Cytoadherence and severe malaria. Malaysian J Med Sci 19: 5-18.

9. Lee W, Russell B, Rénia L, 2019. Sticking for a cause: the falciparum malaria parasites cytoadherence paradigm. Front Immunol 10: 1444.

10. Schofield L, Grau GE, 2005. Immunological processes in malaria pathogenesis. Nat Rev Immunol 5: 722-735.

11. Carvalho BO et al., 2010. On the cytoadhesion of Plasmodium vivax - infected erythrocytes. J Infect Dis 202: 638-647.

12. MacPherson G, Warrell MJ, White NJ, Looareesuwan S, Warrell DA, 1985. Human cerebral malaria - a quantitative ultrastructural analysis of parasitized erythocyte sequestration. $A m \mathrm{~J}$ Pathol 119: 385-401.

13. De Alencar-Filho AC, Vinícius M, De Lacerda G, Okoshi K, Okoshi MP, 2014. Review article malaria and vascular endothelium. Arq Bras Cardiol 103: 165-169.

14. Mohsen A, Green S, West JN, McKendrick M, 2001. Myocarditis associated with Plasmodium falciparum malaria: a case report and a review of the literature. J Travel Med 8: 219-222.

15. Wennicke KF, Wichmann D, Brattig NW, Pankuweit S, Maisch B, Schwarz RT, Ruppert V, 2008. Glycosylphosphatidylinositolinduced cardiac myocyte death might contribute to the fatal outcome of Plasmodium falciparum malaria. Apoptosis 13: 857-866.

16. Moxon CA et al., 2014. Persistent endothelial activation and inflammation after Plasmodium falciparum infection in Malawian children. J Infect Dis 209: 610-615.

17. Hotamisligil GS, 2006. Inflammation and metabolic disorders. Nature 444: 860-867.

18. Rose NR, 2011. Critical cytokine pathways to cardiac inflammation. J Interf Cytokine Res 31: 705-710.

19. World Health Organization, 2015. WHO - Guidelines for the Treatment of Malaria, 3rd edition. Geneva, Switzerland: WHO.

20. World Health Organization, 2017. WHO Evidence Review Group Meeting, October 13-14, 2016. Geneva, Switzerland: Varemebé Conference Centre, 13-14.

21. White NJ, 2007. Cardiotoxicity of antimalarial drugs. Lancet Infect Dis 7: $549-558$.

22. Germany GH, Germany AK, Lenzen MJ, Denmark EP, Vranckx P, 2017. 2017 ESC guidelines for the management of acute myocardial infarction in patients presenting with ST-segment elevation. Eur Hear J 39: 119-177.

23. Task A et al., 2015. 2015 ESC guidelines for the diagnosis and management of pericardial diseases; the task force for the diagnosis and management of pericardial diseases of the European Society of Cardiology. Eur Hear J 36: 2921-2964.

24. Morrow DA, Cannon CP, Jesse RL, Newby LK, Ravkilde J, Storrow AB, Wu AH, Christenson RH; National Academy of Clinical Biochemistry, 2007. National academy of clinical biochemistry laboratory medicine practice guidelines: clinical characteristics and utilization of biochemical markers in. Circulation 53: 356-375.

25. Lang RM et al., 2015. Recommendations for cardiac chamber quantification by echocardiography in Adults: an update from the American Society of Echocardiography and the European Association of Cardiovascular Imaging. J Am Soc Echocardiogr 28: 1-39.e14.

26. Singh V, Martinezclark P, Pascual M, Shaw ES, Neill WWO, 2010. Cardiac biomarkers - the old and the new: a review. Coron Artery Dis 21: 244-256.

27. McCullough PA, Kluger AY, 2018. Interpreting the wide range of NT-proBNP concentrations in clinical decision making. J Am Coll Cardiol 71: 10-12.

28. NIH, 2020. Study Quality Assessment Tools | National Heart, Lung, and Blood Institute (NHLBI). Available at: https:// www.nhlbi.nih.gov/health-topics/study-quality-assessmenttools. Accessed October 30, 2020.

29. Nyaga VN, Arbyn M, Aerts M, 2014. Metaprop: a stata command to perform meta-analysis of binomial data. Arch Public Heal 72: 39.

30. Egger M, Smith GD, Schneider M, Minder C, 1997. Bias in metaanalysis detected by a simple, graphical test. BMJ 315: 629-634.

31. Bhardwaj N, Ahmed Z, Sharma S, Srivastava B, Pande V, 2020. Infection, genetics and evolution clinicopathological study of potential biomarkers of Plasmodium falciparum malaria severity and complications. Infect Genet Evol 77: 104046.

32. Ray HN, Doshi D, Rajan A, Singh AK, Singh SB, Das MK, 2017. Cardiovascular involvement in severe malaria: a prospective study in. J Vector Borne Dis 54: 177-182.

33. Alencar-Filho AC, Marcos Ferreira JMBB, Salinas LJ, Fabbri C, Monteiro W, Siqueira AM, Okosh K, Lacerda MVG, Okoshi MP, 2016. Cardiovascular changes in patients with non-severe Plasmodium vivax malaria. Int J Cardiol Heart Vasc 11: 12-16.

34. Nayak KC, Meena SL, Gupta BK, Kumar S, Pareek V, 2013. Cardiovascular involvement in severe vivax and falciparum malaria. J Vector Borne Dis 50: 285-291.

35. Stauga S, Hahn A, Brattig NW, Fischer-herr J, Baldus S, Burchard GD, Cramer JP, 2013. Clinical relevance of different biomarkers in imported Plasmodium falciparum malaria in adults: a case control study. Malar J 12: 246

36. Herr J, Mehrfar P, Schmiedel S, Wichmann D, Brattig NW, Burchard GD, Cramer JP, 2011. Reduced cardiac output in imported Plasmodium falciparum malaria. Malar J 10: 160.

37. Mehmood A, Ejaz K, Ahmed T, 2012. Original article severity of Plasmodium vivax malaria in Karachi: a cross-sectional study. $J$ Infect Dev Ctries 6: 664-670.

38. Jain K, Chakrapani M, 2010. Acute myocardial infarction in a hospital cohort of malaria. $J$ Glob Infect Dis 2: 72-73.

39. Ehrhardt S, Wichmann D, Hemmer CJ, Burchard GD, Brattig NW, 2004. Circulating concentrations of cardiac proteins in complicated and uncomplicated Plasmodium falciparum malaria. Trop Med Int Heal 9: 1099-1103.

40. Günther A, Grobusch MP, Slevogt H, Abel W, Burchard GD, 2003. Short communication: myocardial damage in falciparum malaria detectable by cardiac troponin T is rare. Trop Med Int Heal 8: 30-32.

41. Mohapatra MK, Mohanty NK, Das SP, 2000. Myocardial injury: an unrecognized complication of cerebral malaria. Trop Doct 30: 188-189.

42. Franzen D, Curtius J, Heitz W, Höpp H, Diehl V, Hilger D, 1992. Clinical investigator cardiac involvement during and after malaria. Clin Investig 70: 670-673.

43. Nguah SB, Hoffmann S, Herr J, Cramer JP, 2012. Cardiac function in Ghanaian children with severe malaria. Intensiv Care Med 38 : 2032-2041.

44. Mocumbi AO, Songane M, Salomao C, Ulibarri R, Ferreira MB, Yacoub MH, 2011. Lack of evidence of myocardial damage in children with Plasmodium falciparum severe and complicated malaria from an endemic area for endomyocardial fibrosis. J Trop Pediatr 57: 312-314.

45. Murphy S, Cserti-gazdewich C, Dhabangi A, Musoke C, Nabukeera-barungi N, Paed MM, King ME, Romero J, Noviski N, Dzik W, 2011. Ultrasound findings in Plasmodium falciparum malaria: a pilot study. Pediatic Crit Care Med 12: e58-e63.

46. Janka JJ et al., 2011. Increased pulmonary pressures and myocardial wall stress in children with severe malaria. $J$ Infect Dis 202: 791-800.

47. Ehrhardt S, Mockenhaupt FP, Anemana SD, Otchwemah RN, Wichmann D, Cramer JP, Bienzle U, Burchard GD, Brattig NW, 2005. High levels of circulating cardiac proteins indicate cardiac impairment in African children with severe Plasmodium falciparum malaria. Microbes Infect 7: 1204-1210.

48. Bhat S, Kumar M, Alva J, 2013. Malaria and the conducting system of the heart. BMJ Case Rep 2013: bcr2012007462.

49. Chandra S, Chandra H, 2011. Myocardial infarction associated with Plasmodium falciparum malarial infection. Ind Med Gaz 2011: 374-375.

50. Colomba C, Trizzino M, Gioè C, Coelho F, Lopo I, Pinheiro P, Sousa J, Cascio A, 2017. Malaria and the heart: two rare case 
reports of Plasmodium falciparum - associated pericarditis. $J$ Vector Borne Dis 54: 372-374.

51. Costenaro P, Benedetti P, Facchin C, Mengoli C, Pellizzer G, 2011. Fatal myocarditis in course of Plasmodium falciparum infection: case report and review of cardiac complications in malaria. Case Rep Med 2011: 202083.

52. Cox-singh J et al., 2010. Severe malaria - a case of fatal Plasmodium knowlesi infection with post-mortem findings: a case report. Malar J 9: 10.

53. Dev N, Gadpayle AK, Sankar J, Choudhary M, 2014. Case report an unusual case of heart failure due to Plasmodium vivax infection with a favorable outcome. Rev Soc Bras Med Trop 47: 663-665.

54. Gantait K, Nayak IG, 2013. Vivax malaria complicated by myocarditis. J Assoc Physicians India 61: 2012-2013.

55. Genrich GL, Guarner J, Paddock CD, Shieh W, Greer PW, Barnwell JW, Zaki SR, 2007. Fatal malaria infection in travelers: novel immunohistochemical assays for the detection of Plasmodium falciparum in tissues and implications for pathogenesis. Am J Trop Med Hyg 76: 251-259.

56. Gupta N, Sahoo SK, 2013. Plasmodium vivax induced myocarditis: a rare case report. Indian J Med Microbiol 31: 180-182.

57. Jain D, Nand N, Verma P, Saxena D, Jain P, 2018. Malaria causing cardiomyopathy and thrombotic microangiopathy: a rare association. Erciyes Med J 40: 237-239.

58. Khan FY, 2019. Imported Plasmodium vivax malaria complicated by reversible myocarditis. J Fam Community Med 26: 232-234.

59. Khattak A, Ali W, Samore N, Idris M, Khan M, Pasha W, 2014. Complicated Plasmodium falciparum malaria initially presenting as myocarditis. J Ayub Med Coll Abbottabad 26: 413-415.

60. Kim SA, Kim ES, Rhee MY, Choi SII, Huh HJ, Chae SL, 2008. A case of myocarditis associated with Plasmodium vivax. J Travel Med 16: 138-140.

61. Kumar P, Kumar CD, Shaik FAR, Ghanta SB, 2010. Case report myocardial dysfunction in severe falciparum malaria. $J$ Trop Pediatr 56: 67-69.

62. Maheshwari M, Resident $Y$, 2012. Plasmodium vivax malaria complicated by pericardial effusion. Ind Med Gaz 5: 199-201.

63. Martins AC, Lins JB, Santos LMN, Fernandes LN, Malafronte RS, Maia TC, Ribera MC, Ribera RB, da Silva-Nunes M, 2014. Vivax malaria in an Amazonian child with dilated cardiomyopathy. Malar J 13: 61.

64. Menezes RG, Kanchan T, Rai S, Rao PPJ, Naik R, Shetty BSK, Lobo SW, Chauhan A, Shetty M, Mathai AM, 2010. An autopsy case of sudden unexplained death caused by malaria. J Forensic Sci 55: 835-838.

65. Miller MJ, Marcus DM, Cameron DC, 1965. Latent infections with plasmodium ovale malaria. Can Med Assoc J 92: 1241-1247.

66. Nieman $A$, de Mast $Q$, Roestenberg M, Wiersma J, Pop G, Stalenhoef A, Druilhe P, Sauerwein R, van der Ven A, 2009. Cardiac complication after experimental human malaria infection: a case report. Malar J 8: 277.

67. Ruhela M, Khandelwal G, Gupta S, Bansal A, Gyanchandani N, 2019. Case report falciparum malaria mimicking acute myocardial infarction. J Fam Med Prim Care 8: 308-310.

68. Sonambekar AA, Gupta N, Agarwal MP, Rajpal S, Aggarwal A, 2014. Plasmodium vivax - associated myopericarditis. Eur $J$ Case Rep Intern Med 8: 1-8.

69. Sulaiman H, Ismail MD, Jalalonmuhali M, Atiya N, 2014. Severe Plasmodium falciparum infection mimicking acute myocardial infarction. Malar J 13: 341.

70. van Meer MPA, Bastiaens GJH, Boulaksil M, de Mast $Q$, Gunasekera A, Hoffman SL, Pop G, van der Ven AJ, Sauerwein $\mathrm{RW}, 2014$. Idiopathic acute myocarditis during treatment for controlled human malaria infection: a case report. Malar J 13: 38.

71. Ventura A, Chaves S, Monteiro JC, Sequeira CG, Ohnishi MD, Souza S, Libonati RM, Sousa RC, Souza JM, 2014. Case report myocarditis associated with Plasmodium vivax malaria: a case report. Rev Soc Bras Med Trop 47: 810-813.
72. Wichmann O, Löscher T, Jelinek T, 2003. Fatal malaria in a German couple returning from Burkina Faso. Infect $J$ 31: 2002-2004.

73. Levin ESP, 1954. Vivax malaria case reports. Calif Med Case Rep 81: 87-89.

74. Jones DWC, 1919. Notes on some occasional manifestations of malaria. Lancet 194: 1131-1133.

75. Trampuz A, Jereb M, Muzlovic I, Prabhu RM, 2003. Clinical review: severe malaria. Crit Care 7: 315-323.

76. Bethel DB, Tung P, Xuan C, Phuon T, Nosten F, Wallery D, 1996. Electrocardiographic monitoring in severe falciparum malaria. Trans R Soc Trop Med Hyg 90: 266-269.

77. Spitz S, 1946. The pathology of acute falciparum malaria. Mil Surg 11: 555-572.

78. Lopes SCP et al., 2014. Paucity of Plasmodium vivax mature schizonts in peripheral blood is associated with their increased cytoadhesive potential. J Infect Dis 209: 1403-1407.

79. Gaieski DF, Goldman JD, Holtzman DL, Shoff WH, Shepherd SM, Mehta N, Goyal M, 2013. Case report - algid malaria treated with early goal-directed therapy. Am J Emerg Med 31: 263.e5-263.e10.

80. Gupta BK, Gupta A, Nehra HR, Balotia HR, Meena SL, Kumar S, 2015. Clinical profile and prognostic indicators in adults hospitalized with severe malaria caused by different Plasmodium species. Infect Dis Res Treat 8: 45-50.

81. Brainin $P$ et al., 2019. Malaria infection and risk of incident heart failure: a nationwide cohort study. Eur Hear J 40: ehz745.0312.

82. Hemmer CJ, Georg F, Holst E, Kern P, Chiwakata CB, 2006. Stronger host response per parasitized erythrocyte in Plasmodium vivax or ovale than in Plasmodium falciparum malaria. Trop Med Int Heal 11: 817-823.

83. Val F et al., 2017. Respiratory complications of Plasmodium vivax malaria: systematic review and meta-analysis. Am J Trop Med Hyg 97: 733-743.

84. Andrade BB, Reis-filho A, Souza-neto SM, Clarêncio J, Camargo LMA, 2010. Severe Plasmodium vivax malaria exhibits marked inflammatory imbalance. Malar J 9: 13.

85. Hojo-Souza SN et al., 2017. On the cytokine/chemokine network during Plasmodium vivax malaria: new insights to understand the disease. Malar J 16: 42.

86. Pollack A, Kontorovich AR, Fuster V, Dec GW, 2015. Viral myocarditis-diagnosis, treatment options, and current controversies. Nat Rev Cardiol 12: 670-680.

87. World Health Organization, 2013. Management of Severe Malaria: A Practical Handbook. Geneva, Switzerland: WHO.

88. Brabin B, Dorman E, Beales P, 2000. Severe falciparum malaria. World health organization, communicable diseases cluster. Trans $R$ Soc Trop Med Hyg 94 (Suppl 1): S1-S90.

89. WHO, 1994. Severe and complicated malaria. World health organization, division of control of tropical diseases. Trans $R$ Soc Trop Med Hyg 84 (Suppl 2): 1-65.

90. Roca-feltrer A, Carneiro I, Schellenberg JRMA, 2008. Estimates of the burden of malaria morbidity in Africa in children under the age of 5 years. Trop Med Int Heal 13: 771-783.

91. Mascarello M, Allegranzi B, Angheben A, Anselmi M, Concia E, Laganà S, Manzoli L, Marocco S, Monteiro G, Bisoffi Z, 2008. Imported malaria in adults and children: epidemiological and clinical characteristics of 380 consecutive cases observed in Verona, Italy. J Travel Med 15: 229-236.

92. Rowe JA, Claessens A, Corrigan RA, 2009. Adhesion of Plasmodium falciparum-infected erythrocytes to human cells: molecular mechanisms and therapeutic implications. Expert Rev Mol Med 11: e16.

93. Gallego-delgado J, Walther T, Rodriguez A, 2017. The high blood pressure protection - malaria. HHS Public Access 119: 1071-1075.

94. Etyang AO, Smeeth L, Cruickshank JK, Scott JAG, 2016. The malaria-high blood pressure hypothesis. Circ Res 119: 36-40.

95. Etyang AO et al., 2019. Effect of previous exposure to malaria on blood pressure in Kilifi, Kenya: a Mendelian randomization study. J Am Heart Assoc 8: e011771. 